\title{
Article \\ Characterization of Class V DyP-Type Peroxidase SaDyP1 from Streptomyces avermitilis and Evaluation of SaDyPs Expression in Mycelium
}

\author{
Kanako Sugawara ${ }^{1,2}{ }^{\oplus}$, Toru Yoshida ${ }^{1}{ }^{\circledR}$, Rena Hirashima ${ }^{1}$, Ryoko Toriumi ${ }^{1}$, Hotaka Akiyama ${ }^{1}$, Yurika Kakuta ${ }^{1}$, \\ Yuki Ishige ${ }^{1}$ and Yasushi Sugano ${ }^{1, *}$ \\ 1 Department of Chemical and Biological Sciences, Faculty of Science, Japan Women's University, \\ 2-8-1 Mejirodai, Bunkyo-Ku, Tokyo 112-8681, Japan; sugawarak@fc.jwu.ac.jp (K.S.); \\ yoshidat@fc.jwu.ac.jp (T.Y.); hirashima.jw.tit@gmail.com (R.H.); m1617062tr@ug.jwu.ac.jp (R.T.); \\ hotakaakiyama@icloud.com (H.A.); kakutay999@gmail.com (Y.K.); tiramisu_g5t5a3t2kitty@yahoo.co.jp (Y.I.) \\ 2 Department of Clinical Laboratory Sciences, Faculty of Health Sciences, Nihon Institute of Medical Science, \\ 1276 Shimogawara, Moroyamamachi, Irumagun, Saitama 350-0435, Japan \\ * Correspondence: suganoy@fc.jwu.ac.jp; Tel.: +81-3-5981-3667
}

check for updates

Citation: Sugawara, K.; Yoshida, T.; Hirashima, R.; Toriumi, R.; Akiyama, H.; Kakuta, Y.; Ishige, Y.; Sugano, Y. Characterization of Class V DyP-Type Peroxidase SaDyP1 from Streptomyces avermitilis and Evaluation of SaDyPs Expression in Mycelium. Int. J. Mol. Sci. 2021, 22, 8683. https://doi.org/ $10.3390 /$ ijms 22168683

Academic Editor: Clemente Capasso

Received: 2 June 2021

Accepted: 9 August 2021

Published: 12 August 2021

Publisher's Note: MDPI stays neutral with regard to jurisdictional claims in published maps and institutional affiliations.

Copyright: (c) 2021 by the authors. Licensee MDPI, Basel, Switzerland. This article is an open access article distributed under the terms and conditions of the Creative Commons Attribution (CC BY) license (https:// creativecommons.org/licenses/by/ $4.0 /)$.

\begin{abstract}
DyP-type peroxidases are a family of heme peroxidases named for their ability to degrade persistent anthraquinone dyes. DyP-type peroxidases are subclassified into three classes: classes $\mathrm{P}$, I and V. Based on its genome sequence, Streptomyces avermitilis, eubacteria, has two genes presumed to encode class V DyP-type peroxidases and two class I genes. We have previously shown that ectopically expressed SaDyP2, a member of class V, indeed has the characteristics of a DyP-type peroxidase. In this study, we analyzed SaDyP1, a member of the same class V as SaDyP2. SaDyP1 showed high amino acid sequence identity to SaDyP2, retaining a conserved GXXDG motif and catalytic aspartate. SaDyP1 degraded anthraquinone dyes, which are specific substrates of DyPtype peroxidases but not azo dyes. In addition to such substrate specificity, SaDyP1 showed other features of DyP-type peroxidases, such as low optimal $\mathrm{pH}$. Furthermore, immunoblotting using an anti-SaDyP2 polyclonal antibody revealed that SaDyP1 and/or SaDyP2 is expressed in mycelia of wild-type S. avermitilis.
\end{abstract}

Keywords: heme peroxidase; dye-decolorizing peroxidase; DyP-type peroxidase; Streptomyces

\section{Introduction}

Heme peroxidases are conserved among almost all organisms and catalyze various oxidative reactions using hydrogen peroxide as an electron acceptor. They are present in both prokaryotes and eukaryotes and are classified by the peroxidase and oxide-reductase database RedoxiBase (https:/ / peroxibase.toulouse.inra.fr/, accessed on 20 July 2021) into six groups [1,2]: catalases, haloperoxidases, di-heme cytochrome c peroxidases, animal peroxidases, non-animal peroxidases (previously called plant peroxidase [3]) and DyP-type peroxidases. Dye-decolorizing peroxidase (DyP; EC 1.11.1.19), a novel peroxidase isolated from the basidiomycete Bjerkandera adusta Dec 1 [4], is capable of degrading persistent anthraquinone dyes, in contrast with other types of peroxidases [5]. DyP differs from existing heme peroxidases in terms of amino acid sequence, tertiary structure, substrate specificity, catalytic residues and optimum $\mathrm{pH}$. For these reasons, it was originally considered an exceptional peroxidase. However, DyP and a series of genes phylogenetically similar to DyP were proposed to constitute a novel heme peroxidase family, the DyP-type peroxidase because many genes similar to DyP were found, and for those cases in which the corresponding protein has been purified, their biochemical characterizations have been revealed similar to those of DyP [6,7].

Conventionally, RedoxiBase has subdivided DyP-type peroxidases into classes A, $\mathrm{B}, \mathrm{C}$ and $\mathrm{D}$ based on similarity of amino acid sequences [1]. However, although amino 
acid sequences between different classes exhibit low similarity, tertiary structures are highly similar. Therefore, a new classification of the DyP-type peroxidase family has been proposed using structure-based sequence alignments [8]. In this new scheme, classes $\mathrm{P}$ and I correspond to former classes B and A, respectively, whereas former classes C and D are combined into a new class $\mathrm{V}$.

Many enzymes have been annotated as DyP-type peroxidases and characterized based on expression and biochemical properties of purified proteins, establishing DyP-type peroxidases as enzymes with potentially very diverse but still poorly physiological functions. The full range of DyP-type peroxidase roles remains unclear, but it is increasingly thought that these functions may be subclass dependent. Most DyPs from basidiomycetes belong to class V. These include TAP from Termitomyces albuminosus [9], AjPI and AjPII from Auricularia auricula-judae [10], MsP1 and MsP2 from Marasmius scorodonius [11], DyP-type peroxidase from Irpex lacteus [12], MepDyP from Mycena epipterygia [13] and EglDyP from Exidia glandulosa [13], all of which can degrade a lignin, suggesting that they have physiological roles in lignin degradation. We have also recently shown that antimicrobial substances retained by trees that prevent parasitism from fungi are candidate physiological substrates of DyP from B. adusta Dec 1 [14]. However, research on class V enzymes from bacteria is limited to DyP2 from Amycolatopsis sp. [15], AnaPX from Anabaena sp. [16-18] and SaDyP2 from S. avermitilis [19]. The physiological roles of them have not yet been established. Most bacterial DyP-type peroxidases belong to classes $\mathrm{P}$ and I, and are not limited to oxidative degradation of substrates, being capable of performing various functions in the cell. For instance, YfeX and EfeB (YcdB) from Escherichia coli may function as dechelatases, extracting an iron atom from heme without causing tetrapyrrole degradation [20]. However, whether YfeX and EfeB actually function as dechelatases in vivo remains unclear [21]. Furthermore, EfeB is also a component of a ferrous iron transporter [22], and it has been reported that several DyPs are likely to function in iron transport [23-25].

Streptomyces avermitilis is a gram-positive eubacterium whose entire genome has been analyzed [26]. S. avermitilis has four genes that are predicted to encode proteins with amino acid sequences highly similar to those of DyP-type peroxidases. Two of them, SavDyPrx03-1 (SAV_549) and SavDyPrx03-2 (SAV_3599), are deposited as class C DyP-type peroxidases (now class V) in RedoxiBase [1,2]. Using ectopic expression and biochemical characterization, we previously showed that SaDyP2, encoded by SavDyPrx03-2, indeed has features of a DyP-type peroxidase. The remaining two genes, SAV_4242 and SAV_5925, encode enzymes that are predicted to be class A (now class I) DyP-type peroxidases based on a Peroxiscan search in RedoxiBase [1,2]. In the case of SAV_4242, its homolog in S. lividans, DtpA, was reported to function in the formation of aerial mycelium via copper transport [27]. In addition to its function, the crystal structure and enzymatic reaction mechanism of DtpA have been investigated in detail [28-31]. Crystal structures of the enzyme from S. coelicolor have also been registered in the Protein Data Bank (PDB: 4GRC and 4GT2). However, virtually all studies in Streptomyces to date have been on Class I enzymes, with few studies on Class V enzymes. In addition, although S. lividans and S. coelicolor are well-studied Streptomyces, the genes encoding class V DyP-type peroxidases have not been found in either species. In this study, we ectopically expressed the DyP-type peroxidase encoded by SavDyPrx03-1 (SAV_549), termed SaDyP1, and characterized it. In addition, immunoblotting demonstrated the expression of class $V$ enzymes in wild-type S. avermitilis.

\section{Results}

\subsection{Amino Acid Sequences and Structure Alignment}

Whole-genome sequencing showed that $S$. avermitilis has four candidate DyP-type peroxidase genes [26]. Two of these, SavDyPr03-1 (SAV_549) and SavDyPrx03-2 (SAV_3599), encode proteins predicted to be class $V$ (formerly class $C$ in RedoxiBase) that we named SaDyP1 and SaDyP2, respectively [19]. A ClustalW-based amino acid sequence alignment of SaDyP1, SaDyP2 and DyP from B. adusta, a class V DyP-type peroxidase, is shown in 
Figure 1A. The amino acid sequences of SaDyP1 and SaDyP2 are quite similar, exhibiting $59.1 \%$ identity. On the other hand, the sequence identity with DyP is lower for SaDyP1 $(27.2 \%)$ and SaDyP2 (28.3\%). The remaining two genes, SAV_4242 and SAV_5925, are predicted to encode class I (formerly class A) proteins that we named SaDyP3 and SaDyP4, respectively. The amino acid sequence alignments of SaDyP3 and SaDyP4 with EfeB from E. coli, a Class I DyP-type peroxidase, are shown in Figure 1B. The amino acid sequence homology between SaDyP3 and SaDyP4 is 39.7\%. SaDyP3 is a homologue of DtpA, a DyPtype peroxidase from $S$. lividans, and the two peroxidases show an amino acid sequence identity of 78.4\%. The DyP-type peroxidase from Streptomyces coelicolor (PDB: 4GRC) is a SaDyP4 homologue, and these two enzymes exhibit an amino acid sequence identity of $85.6 \%$. On the other hand, genes homologous to those encoding SaDyP1 and SaDyP2 do not exist in well-studied Streptomyces sp. such as S. lividans and S. coelicolor. Structure alignment of SaDyP1 model constructed by SWISS-MODEL and DyP (PDB: 3afv) was shown in Figure 1C. SaDyP1 has conserved aspartate D176, other conserved residues and unique GXXDG motif of DyP-type peroxidases. Comparison of the conserved amino acid residues around the heme, the positions of the residues were in reasonable correspondence between DyP and SaDyP1 models.

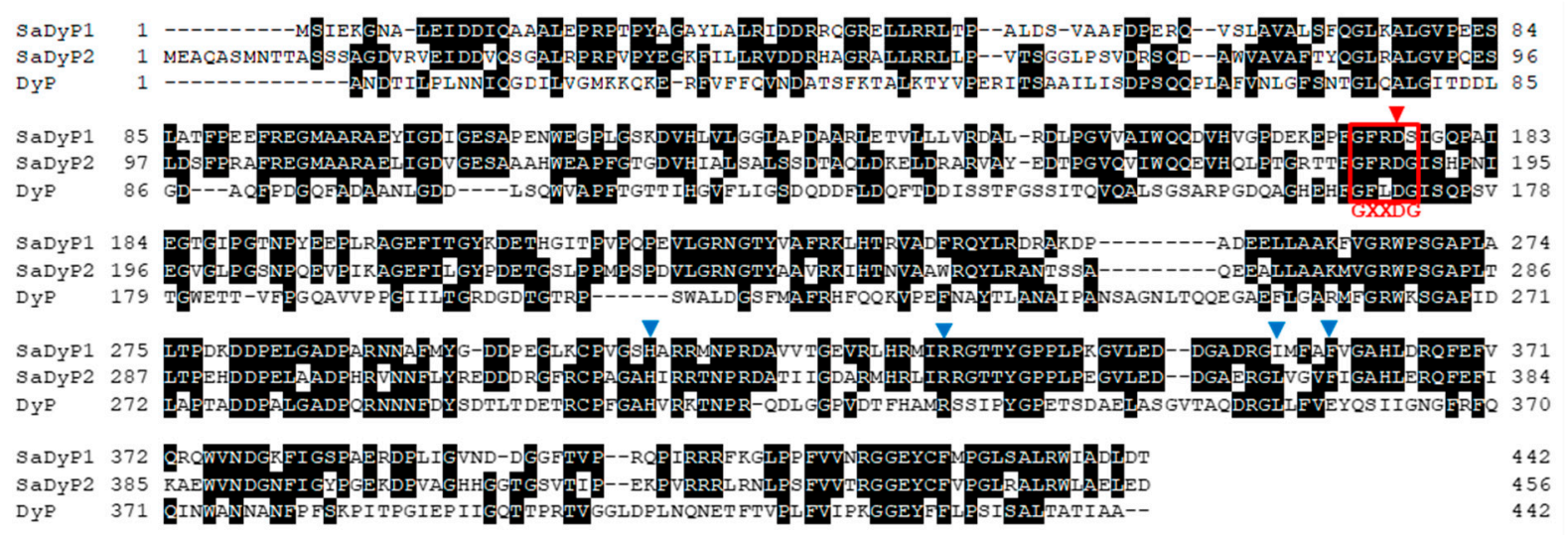

(A)

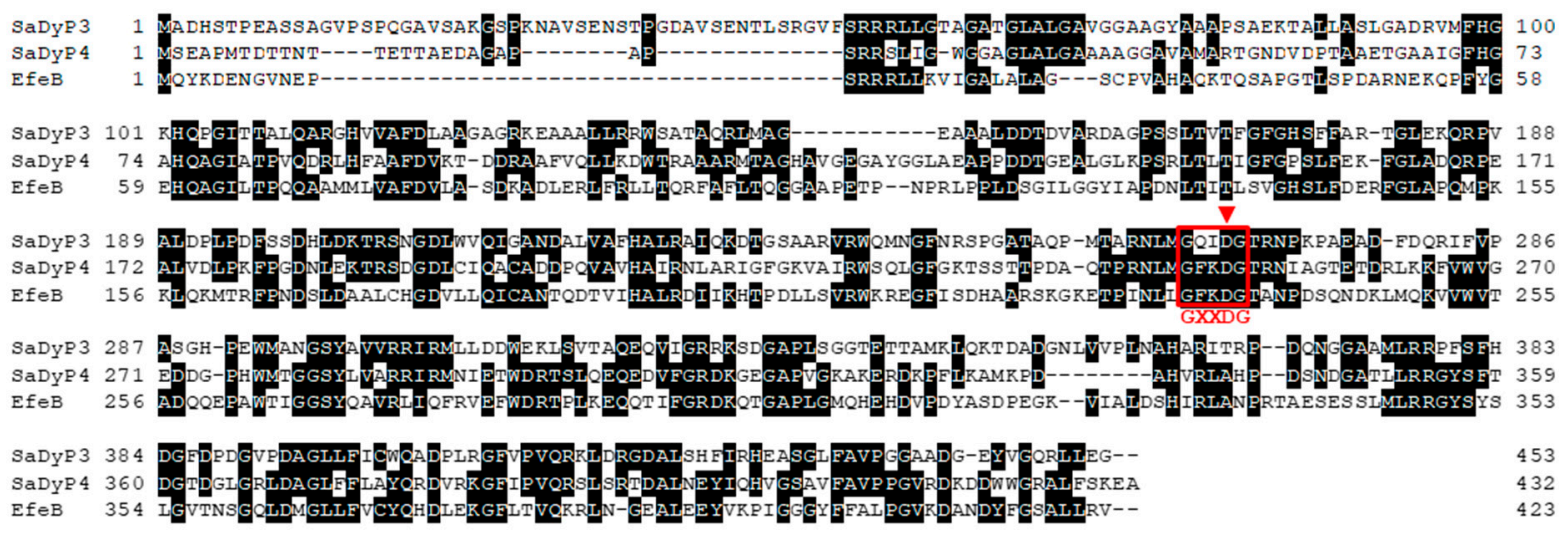

(B)

Figure 1. Cont. 

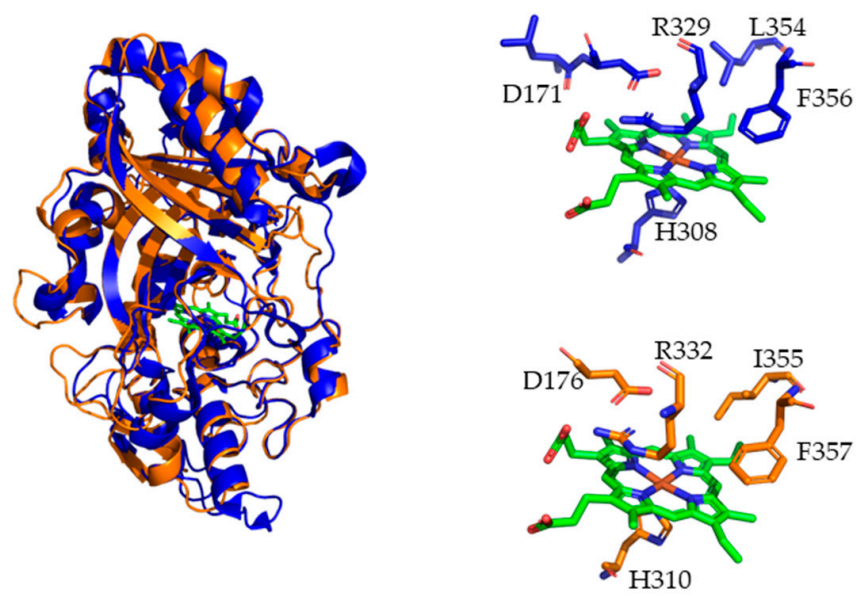

(C)

Figure 1. Amino acid sequence and structure alignments between SaDyPs and representative DyP-type peroxidases. (A) SaDyP1, SaDyP2 and DyP from B. adusta. (B) SaDyP3, SaDyP4 and EfeB. Residues with two or more matches are indicated with black fill. The unique GXXDG motifs of DyP-type peroxidases are surrounded by a red line. Catalytic aspartate of $\mathrm{DyP}$ is indicated by red arrowheads [6]. Blue arrowheads show four amino acid residues that form the hydrogen peroxide binding pocket of DyP [21]. (C) Homology modeling of SaDyP1 using SWISS-MODEL. Orange, the model of SaDyP1; blue, DyP. A model constructed using an ancestral class V DyP-type peroxidase (PDB:7anv) as a template. Left, structure alignment of the model of SaDyP1 and DyP (PDB: 3afv). Upper-right, enlarged view around heme in DyP (PDB: 3afv). Catalytic aspartate and four amino acid residues highly conserved around heme in DyP-type peroxidase were indicated. Bottom, enlarged view around heme in the model of SaDyP1. Amino acid residues of SaDyP1 model corresponding in DyP (upper-right) were shown. The drawing and alignment were by the PyMOL Molecular Graphics System, Version 2.5 Schrödinger, LLC.

\subsection{Purification of $S a D y P 1$}

We previously characterized heterologously expressed SaDyP2 using S. lividans as an expression host [19]. In the current study, we expressed and characterized SaDyP1 in the same manner. SaDyP1, similar to SaDyP2, was not secreted outside of cells [19]. Heterologously expressed heme peroxidases often adopt the apo form, which lacks a heme molecule, but the spectrum of expressed SaDyP1 showed apparent absorbance at $406 \mathrm{~nm}$ (Soret band), indicating incorporation of a heme molecule (Table 1 and Figure 2A). However, Reinheit Zahl (Rz) values suggested the presence of some apo form; thus, we incubated the Ni-NTA eluate with hemin chloride to incorporate heme. After dialysis and size-exclusion chromatography to remove free hemin and non-target protein, we characterized the spectrum and measured peroxidase activity. These analyses showed an increase in $\mathrm{Rz}$ value upon addition of hemin chloride but no increase in peroxidase activity (Table 1); the spectrum of SaDyP1 purified after adding the hemin reagent showed the presence of a shoulder peak around $350 \mathrm{~nm}$ (Figure 2A).

Table 1. Peroxidase activity of SaDyP1 purified with or without hemin using ABTS as a substrate.

\begin{tabular}{ccc}
\hline & Activity $(\mu \mathrm{mol} / \mathbf{m i n} / \mathbf{m g})$ & Rz Value \\
\hline hemin $(-)$ & 1.88 & 1.22 \\
hemin $(+)$ & 1.74 & 1.57 \\
\hline
\end{tabular}

Purified SaDyP1 was resolved by sodium dodecyl sulfate-polyacrylamide gel electrophoresis (SDS-PAGE) and stained with Coomassie brilliant blue (CBB). Two bands appeared nearly $49.4 \mathrm{kDa}$, the predicted molecular mass of SaDyP1 with a $6 \times$ His tag (Figure 2B). The N-terminal amino acid sequence of both bands contained the N-terminus of the SaDyP1 sequence. Therefore, the two bands are both derived from ectopically ex- 
pressed SaDyP1. On the basis of these observations, we chose to use the SaDyP1 purified without hemin chloride for subsequent characterizations.

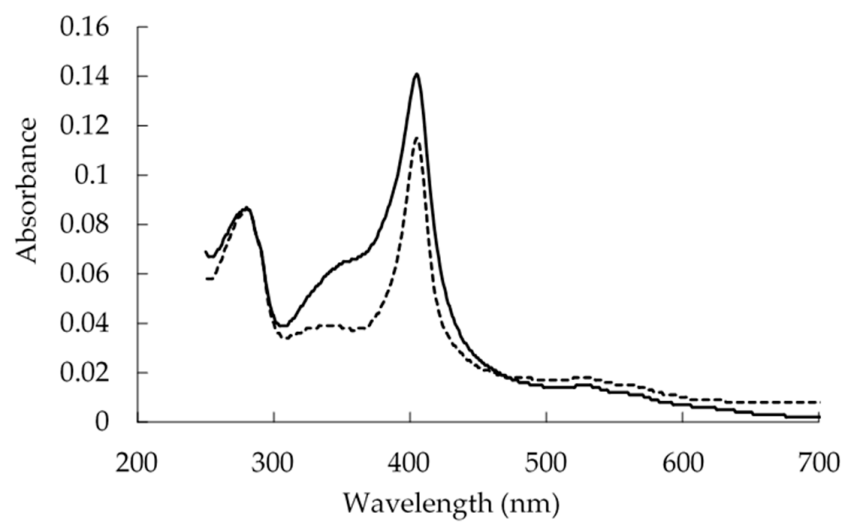

(A)

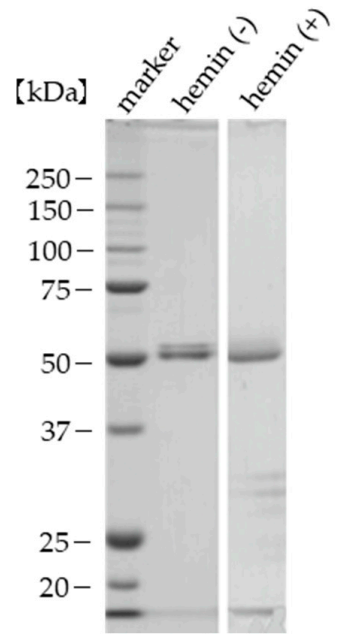

(B)

Figure 2. Analysis of purified SaDyP1, with or without hemin. (A) Electronic absorption spectroscopic characterization of purified SaDyP1, with or without hemin. Solid line, with hemin; dashed line, without hemin. A Soret band was observed at $406 \mathrm{~nm}$. (B) SDS-PAGE analysis of purified SaDyP1, with or without hemin, stained with CBB. SaDyP1 was resolved by electrophoresis on a $10 \%$ polyacrylamide gel and then stained with CBB R-250.

\subsection{Optimal $\mathrm{pH}$ and Thermostability of SaDyP1}

DyP-type peroxidase is characterized by a lower $\mathrm{pH}$ optimum than other heme peroxidase [6,32]. To determine whether SaDyP1 has a lower optimum $\mathrm{pH}$, we measured peroxidase activity against ABTS (2,2'-azinobis [3-ethylbenzothiazoline-6-sulfonic acid]) and 2,6-dimethoxyphenol (DMP) between $\mathrm{pH} 3.0$ and 7.0. SaDyP1 showed the highest peroxidase activity for ABTS at $\mathrm{pH} 4.5$ and for DMP at $\mathrm{pH} 4.0$ (Figure 3), essentially the same behavior as that shown by SaDyP2 [19]. To examine $\mathrm{pH}$ profile for anthraquinone dye, peroxidase activity against Acid Blue 324 was also measured between $\mathrm{pH} 3.5$ and 5.5. SaDyP1 showed the highest activity for Acid Blue 324 at $\mathrm{pH}$ 4.5. Differently from those for ABTS and DMP, the degradative activity of SaDyP1 for Acid Blue 324 was almost undetectable, except at $\mathrm{pH} 4.5$.

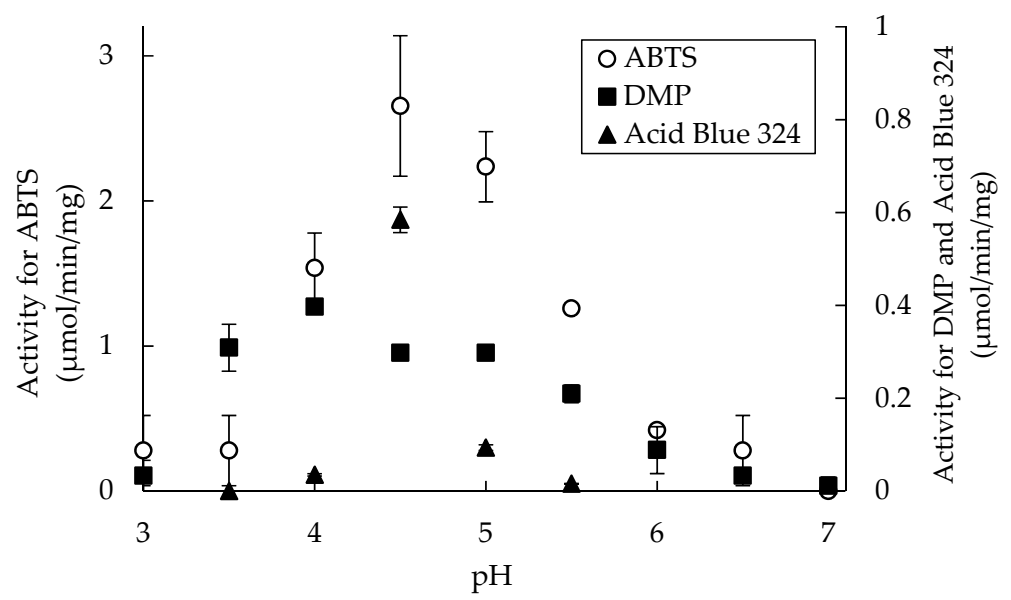

Figure 3. Optimal $\mathrm{pH}$ for the peroxidase activity of SaDyP1. Peroxidase activities against ABTS and DMP were measured between $\mathrm{pH} 3$ and 7. Decolorizing activities against Acid Blue 324 were measured between pH 3.5 and $5.5(n=3)$. Open circle, ABTS; closed square, DMP; closed triangles, Acid Blue 324. 
The thermostability of SaDyP1 activity was measured at $30{ }^{\circ} \mathrm{C}$ after treatment at various temperatures (Figure $4 \mathrm{~A}, \mathrm{~B}$ ). SaDyP1 retained almost all of its initial activity against ABTS and DMP after incubation at $30{ }^{\circ} \mathrm{C}$ or $40{ }^{\circ} \mathrm{C}$ for $2 \mathrm{~h}$ (Figure $4 \mathrm{~A}, \mathrm{~B}$ ). The peroxidase activity of SaDyP1 against ABTS significantly decreased after incubating at $50{ }^{\circ} \mathrm{C}$ for $1 \mathrm{~h}$ and was almost completely lost following incubation at $60^{\circ} \mathrm{C}$ for $30 \mathrm{~min}$ (Figure $4 \mathrm{~A}$ ). On the other hand, the activity of SaDyP1 against DMP was maintained at $50{ }^{\circ} \mathrm{C}$.

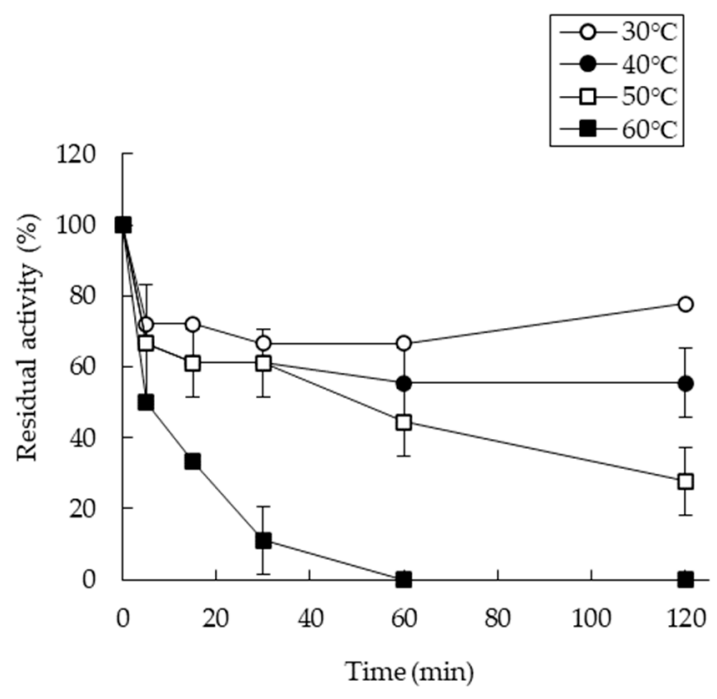

(A)

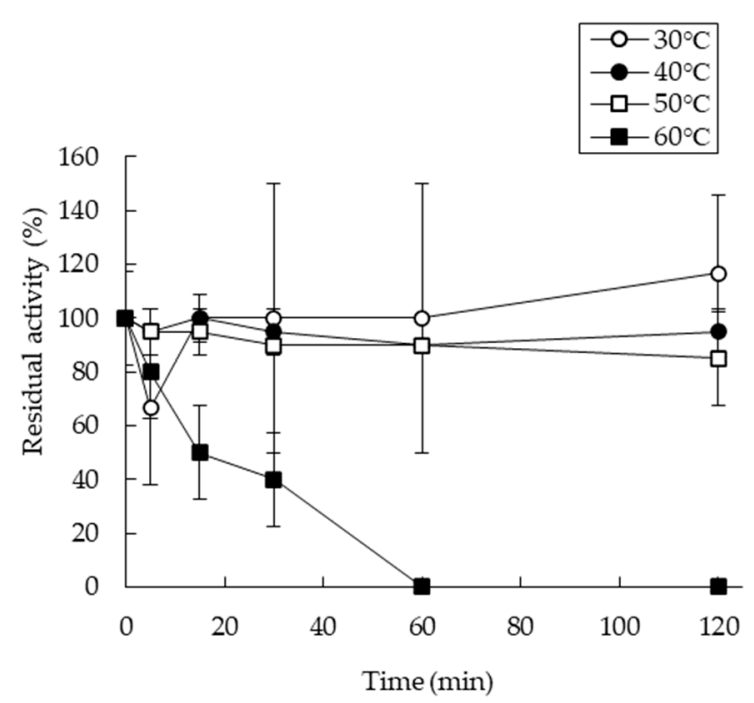

(B)

Figure 4. Thermostability of purified SaDyP1. SaDyP1 peroxidase activity toward ABTS (A) and DMP (B) at $30{ }^{\circ} \mathrm{C}$ after incubation at $30^{\circ} \mathrm{C}, 40{ }^{\circ} \mathrm{C}, 50^{\circ} \mathrm{C}$ or $60^{\circ} \mathrm{C}(n=3)$. Residual activity at $0 \mathrm{~h}$ was defined as $100 \%$. Open circles, $30{ }^{\circ} \mathrm{C}$; closed circles, $40^{\circ} \mathrm{C}$; open squares, $50{ }^{\circ} \mathrm{C}$; closed squares, $60{ }^{\circ} \mathrm{C}$.

\subsection{Substrate Specificity and Catalytic Properties}

Class V DyP-type peroxidases are characterized by their ability to efficiently decolorize anthraquinone dyes, but not azo dyes. SaDyP1 is predicted to be a class V enzyme (formerly class C) based on amino acid sequence homology [1,2]. To assess the selectivity of the decolorizing activity of SaDyP1, we tested several anthraquinone and azo dyes as substrates. SaDyP1 decolorized the anthraquinone dye Acid Blue 324, but was inactive against the anthraquinone dyes AQ-2, M303 and all azo dyes tested (Table 2). In addition, the decolorizing activity against Acid Blue 324 was lower than that of DyP, which belongs to the same class $\mathrm{V}$ [33]. These results indicate that SaDyP1 belongs to class V, based on its degradative activity against anthraquinone dyes, although its activity is lower than that of most class V DyP-type peroxidases.

Table 2. Dye-decolorizing activity of purified SaDyP1 toward anthraquinone or azo.

\begin{tabular}{ccccc}
\hline & Chromophore & $\begin{array}{c}\varepsilon \text { at } \boldsymbol{\lambda} \text { Max } \\
(\mathbf{n m})\end{array}$ & $\begin{array}{c}\text { Initial Conc. } \\
(\boldsymbol{\mu M})\end{array}$ & $\begin{array}{c}\text { Decolorizing Rate } \\
(\boldsymbol{\mu m o l} / \mathbf{m i n} / \mathbf{m g})\end{array}$ \\
\hline Acid Blue 324 & $\mathrm{AQ}$ & 608 & 200 & 1.02 \\
AQ-2 & $\mathrm{AQ}$ & 600 & 100 & N.D. \\
M303 & $\mathrm{AQ}$ & 476 & 100 & N.D. \\
Reactive Black 5 & $\mathrm{AZ}$ & 598 & 20 & N.D. \\
Reactive Red 33 & $\mathrm{AZ}$ & 500 & 20 & N.D. \\
\hline
\end{tabular}

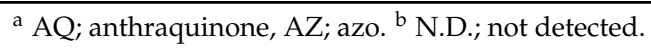

To determine the kinetic parameters of SaDyP1 against ABTS, DMP and Acid Blue 324, we determined substrate saturation curves for these typical peroxidase substrates 
(Figure 5). The peroxidase activity of SaDyP1 toward ABTS increased in a substrate concentration-dependent manner up to $5 \mathrm{mM}$ but decreased above $10 \mathrm{mM}$ probably with substrate inhibition. Therefore, we fitted the activity for ABTS to the Haldane equation describing substrate inhibition kinetics [34] and calculated $k_{\text {cat }}, K_{\mathrm{m}}$ and $K_{\mathrm{i}}$ (Table 3). The concentration-dependent activities with DMP were fitted to the Michaelis-Menten curve and the values of $k_{\text {cat }}$ and $K_{\mathrm{m}}$ were calculated (Table 3). Then, concentration-dependent activity of Acid Blue 324, anthraquinone substrate, was plotted and a sigmoidal curve fitted to Hill equation was obtained (Hill coefficient $=4.1$ ). The $K_{\mathrm{m}}$ values of SaDyP1 are similar to those previously reported for B. adusta DyP against ABTS and DMP [35]. In contrast, the $k_{\text {cat }}$ values of SaDyP1 for ABTS and DMP were much lower than those for DyP. These results suggest that $\mathrm{SaDyP} 1$ is less capable of oxidizing typical peroxidase substrates than other class $\mathrm{V}$ enzymes.

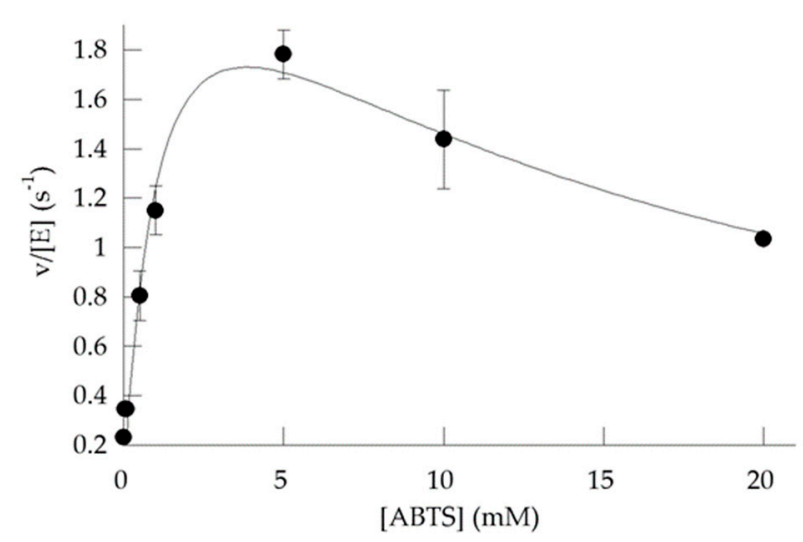

(A)

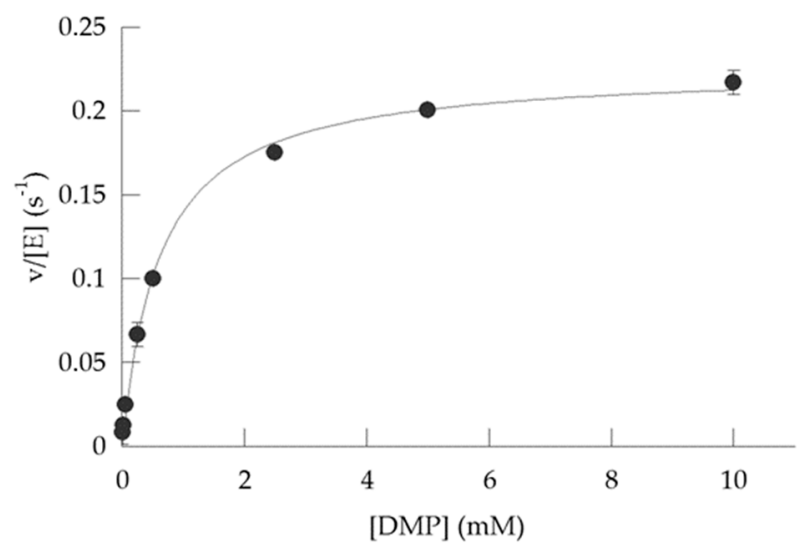

(B)

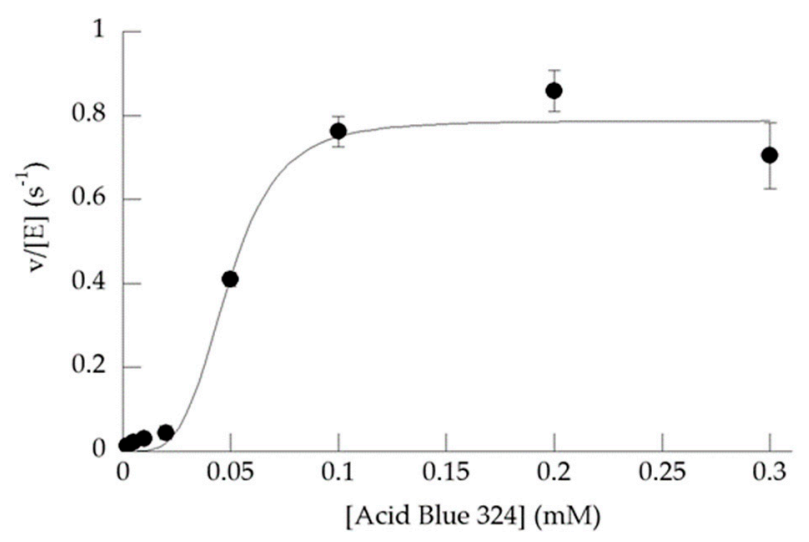

(C)

Figure 5. Substrate saturation curves of SaDyP1 for (A) ABTS at pH 4.5, (B) DMP at pH 4.0 and (C) Acid Blue 324 at pH 4.5 $(n=3)$. The plots were fitted to Haldane equation (ABTS), Michaelis-Menten equation (DMP) and Hill equation (Acid Blue 324).

Table 3. Kinetic parameters of purified SaDyP1 toward several substrates.

\begin{tabular}{cccccc}
\hline & $\mathbf{p H}$ & $\boldsymbol{K}_{\mathbf{m}}(\mathbf{m M})$ & $k_{\text {cat }}\left(\mathbf{s}^{-\mathbf{1}}\right)$ & $\boldsymbol{K}_{\mathbf{i}}(\mathbf{m M})$ & $k_{\text {cat }} / \boldsymbol{K}_{\mathbf{m}}\left(\mathbf{M}^{-\mathbf{1}} \mathbf{s}^{-\mathbf{1}}\right)$ \\
\hline ABTS & 4.0 & 1.2 & 2.8 & 13 & $2.4 \times 10^{3}$ \\
DMP & 4.0 & 0.61 & 0.23 & - & $3.8 \times 10^{2}$ \\
Acid Blue 324 & 4.5 & 0.049 & 0.79 & - & $1.6 \times 10^{4}$ \\
\hline
\end{tabular}




\subsection{Expression of SaDyPs in Wild-Type S. avermitilis Mycelia}

To determine whether SaDyP is expressed in wild-type S. avermitilis, we performed immunoblotting using a polyclonal antibody against SaDyP2, generated using a purified $\mathrm{SaDyP} 2$ that was previously ectopically expressed in S. lividans. First, we examined the reactivity of the anti-SaDyP2 antibody against recombinant SaDyP1 expressed in S. lividans and SaDyP4 expressed in E. coli. SaDyP1 cross-reacted with anti-SaDyP2, but not SaDyP4 (Figure 6A, right), an outcome that is not entirely surprising given the high amino acid sequence homology between SaDyP1 and SaDyP2 (Figure 1A). Next, cultured mycelia of wild-type S. avermitilis were disrupted, and proteins in the resulting supernatant were resolved by SDS-PAGE and immunoblotted using a SaDyP2 antibody. This immunoblot analysis revealed SaDyP2 antibody-reactive band (Figure 6B). Given the cross-reactivity shown in Figure 6A and the molecular weight estimated from the mobility on SDS-PAGE gels, the observed bands can be presumed to be SaDyP1 and/or SaDyP2. These results indicate that at least one of these class V SaDyPs is indeed expressed in S. avermitilis.

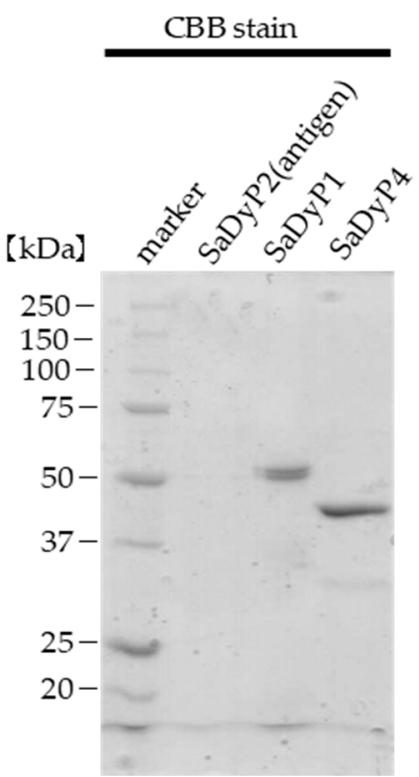

(A)

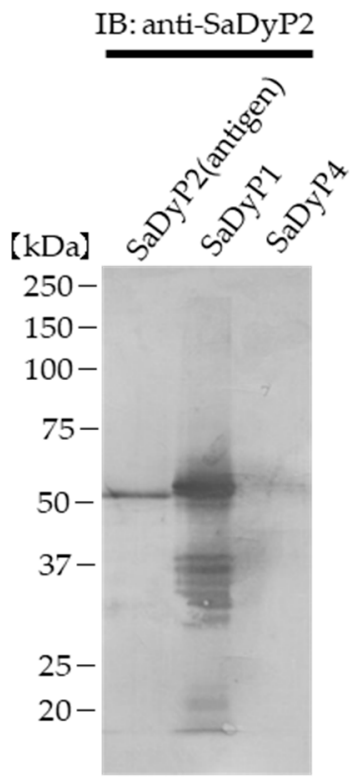

Figure 6. Cross-reactivity of an anti-SaDyP2 polyclonal antibody toward recombinant SaDyP1 and SaDyP4. (A) SDS-PAGE of SaDyP2 (antigen), SaDyP1 and SaDyP4, showing staining with CBB-G250 (left) and immunoreactivity to the anti-SaDyP2 antibody. (B) SDS-PAGE of S. avermitilis mycelium followed by immunostaining with anti-SaDyP2. sup., supernatant; ppt., precipitate.

\section{Discussion}

S. avermitilis is predicted to have four genes encoding DyP-type peroxidases. Since most bacterial DyP-type peroxidases are classified as classes P or I, SaDyP1 and SaDyP2 are expected to exert unique functions in S. avermitilis. SaDyP3 and SaDyP4 are predicted to belong to class I based on assignment to former class A by Peroxiscan, a program that facilitates the subclassification of amino acid sequences in the RedoxiBase. DtpA, a homologue of SaDyP3 in S. lividans, was recently proposed to function in the copperdependent morphogenesis pathway [27]. SaDyP4 is very similar in amino acid sequence to EfeB (Figure 1A). On the other hand, the functions and characteristics of the homologs of SaDyP1 and SaDyP2 have not been reported. A BlastP search revealed that Streptomyces hirsutus contains an enzyme that is $73.0 \%$ identical in amino acid sequence to SaDyP1, and enzymes similar to SaDyP2 are found in several Streptomyces species, including Streptomyces mirabilis (95.3\% identity), Streptomyces olivochromogenes (93.8\%) and S. hirsutus (93.2\%). However, compared with these Streptomyces, for which the few reports of gene disruption, 
a gene disruption method in S. avermitlis has been established [36], being suitable for clarifying the physiological function of class V DyP peroxidases from Streptomyces.

DyP peroxidase lack the distal histidine as an acid-base catalyst, and we have previously proposed that DyP utilizes aspartate instead [6,32]. The alignment of the amino acid sequences and structures of SaDyP1, SaDyP2 and DyP shown in Figure 1A,C showed that the distal aspartate and GXXDG motif are conserved in SaDyP1. However, Mendes et al. reported that the aspartate and arginine are important for the proper binding of $\mathrm{H}_{2} \mathrm{O}_{2}$ to the heme, but none is individually indispensable for promoting $\mathrm{H}_{2} \mathrm{O}_{2}$ (de)protonation and $\mathrm{O}-\mathrm{O}$ bond cleavage in BsDyP from Bacillus subtilis [37]. D235A and R347E mutations in EfeB from Escherichia coli O157 inactivated the enzyme, but D235N retained its activity [38]. In DyPB from Rhodococcus jostii RHA1 and PpDyP from Pseudomonas putida MET94, the substitution of distal arginine, not of aspartate, results in a decrease in their activities $[39,40]$. Thus, it is a controversial issue, which residue functions as an acid-base catalyst in DyP-type peroxidase.

In most cases of recombinant expression of hemoproteins using E. coli as a host, the expressed proteins lack the cofactor heme. In these situations, heme is incorporated by adding 5-aminolevulinic acid, a precursor of heme, to the culture medium or by adding hemin chloride in vitro after expression. On the other hand, when we used Aspergillus oryzae as a host for the recombinant expression of DyP, the holoenzyme was expressed without any additional manipulation [5]. In the current study, a part of SaDyP1 recombinantly expressed in S. lividans as a host incorporated heme without any extrinsic addition and showed peroxidase activity (Table 1 and Figure 3). On the other hand, the Rz value of SaDyP1 increased upon addition of hemin chloride, suggesting that a percentage of apoenzymes incorporate heme. Interestingly, the peroxidase activity did not increase with the addition of hemin chloride, despite evidence of additional incorporation of heme into the apoenzyme. We hypothesized two reasons: (1) Free hemin, which could not be removed by dialysis or ultrafiltration, causing an apparent increase in $\mathrm{Rz}$ value and the additional peak at $350 \mathrm{~nm}$. (2) Heme was incorporated into the inappropriate site of SaDyP1, causing the shoulder peak. In any case, since the addition of hemin did not increase the peroxidase activity, purification without hemin was adopted in this study. Since the expression efficiency of the pHSA81 vector and S. lividans expression system was comparable to that of the $E$. coli expression system, the observation that the $S$. lividans system incorporated much more heme than the E. coli system was unexpected. This greater efficiency of the S. lividans system, despite ectopic expression in both cases, may be attributable to the fact that the target protein and the expression host are of the same species. There are few reports for recombinant expression of heme proteins by pHSA81, and the incorporation of heme into heme proteins in ectopically expression is still under investigation.

SaDyP1 degraded the anthraquinone dye Acid Blue 324 but was inactive against the anthraquinone dyes AQ-2, M303 and all azo dyes tested (Table 2). These results are consistent with the unique features of DyP-type peroxidase, which is capable of degrading persistent anthraquinone compounds. $k_{\text {cat }} / K_{\mathrm{m}}$ values of SaDyP1 for Acid Blue 324 were $1.61 \times 10^{3} \mathrm{M}^{-1} \mathrm{~s}^{-1}$ (Figure 5C and Table 3). SaDyP2 exhibited $k_{\text {cat }} / K_{\mathrm{m}}$ value of $1.24 \mathrm{M}^{-1} \mathrm{~s}^{-1}$ for Acid Blue 324 in our previous report [19]. By comparison, DyP and AjPI from A. auriculajudae showed $k_{\text {cat }} / K_{\mathrm{m}}$ values of $10^{7}$ and $10^{6}$, respectively $[8,10]$. AnaPX from Anabaena sp. and DyP2 from Amycolatopsis sp. 75iv2 - two prokaryotic enzymes belonging to class $\mathrm{V}$-exhibited $k_{\text {cat }} / K_{\mathrm{m}}$ values of $10^{7}$ and $10^{5} \mathrm{M}^{-1} \mathrm{~s}^{-1}$, respectively [15,17]. These results showed that the $k_{\text {cat }} / K_{\mathrm{m}}$ of SaDyP1 and SaDyP2 for anthraquinone compounds were low. On the other hand, the $K_{\mathrm{m}}$ values of SaDyP1 and SaDyP2 for Acid Blue 324 were similar to those of B. adusta DyP. Thus, the lower $k_{\mathrm{cat}} / K_{\mathrm{m}}$ of SaDyP1 and SaDyP2 was accounted for the lower $k_{\text {cat }}$. Even though a class I enzyme, the $k_{\text {cat }}$ value of DtpA from S. lividans for Reactive Blue 19 was on the order of $10^{-2}$ to $10^{-1}$ estimated from the previous study [29], which is lower than that of other Class I enzymes such as TfuDyP $\left(k_{\text {cat }}=10 \mathrm{~s}^{-1}\right)$, DyPA $\left(k_{\mathrm{cat}}=13 \mathrm{~s}^{-1}\right), \operatorname{BsDyP}\left(k_{\mathrm{cat}}=7.9 \mathrm{~s}^{-1}\right)$ [40-42]. As a reason why DyPs from Streptomyces show low degrading activities of some anthraquinone compounds is unknown, clarifying 
the basis for differences in decolorizing activities among enzymes belonging to class $\mathrm{V}$ will require more studies on class $\mathrm{V}$ enzymes from both prokaryotes and eukaryotes. One of the most powerful tools for such investigations is tertiary structural analysis, which enables a detailed discussion of the active site. Therefore, we consider the determination of the tertiary structure of SaDyP1 as a future challenge.

DyP-type peroxidase is characterized by a lower $\mathrm{pH}$ optimum than other heme peroxidases. In this study, SaDyP1 actually showed a low $\mathrm{pH}$ optimum. It has been thought that the low $\mathrm{p} K_{\mathrm{a}}$ value of aspartate contributes to the low $\mathrm{pH}$ optimum [6,32]. However, the $\mathrm{p} K_{\mathrm{a}}$ value of amino acid residue buried into the protein is generally different from the $\mathrm{p} K_{\mathrm{a}}$ value of free amino acid. Recently, Uchida et al. reported that radical transfer but not aspartate is essential for the low $\mathrm{pH}$ optimum [43]. A hydrogen bond located on a potential radical transfer route is responsible for the low $\mathrm{pH}$ optimum. The optimal $\mathrm{pH}$ values for SaDyP1 peroxidase activity against ABTS and DMP- $\mathrm{pH} 4.5$ and 4.0, respectively - were exactly the same as those for SaDyP2 (Figure 3). These pH optima for SaDyP1 and SaDyP2 are slightly higher than the optimal pH for DyP (pH 3.2). SaDyP1 was thermostable at higher temperatures, retaining $\sim 30 \%$ or more of its initial activity against ABTS and DMP even after incubation at $50{ }^{\circ} \mathrm{C}$ for $2 \mathrm{~h}$. DyP has been reported to be thermostable compared with horseradish peroxidase [4], and its stability is contributed by $\mathrm{N}$-linked glycosylations at three Asn residues located on DyP surface. On the other hand, it is unlikely that SaDyP1 from the prokaryote $S$. avermitilis is glycosylated, so the basis for the stability of SaDyP1 at $50{ }^{\circ} \mathrm{C}$ remains uncertain. Four genes from the genome of S. avermitilis have been annotated as DyP-type peroxidases, but to date it remains unclear whether all four are actually expressed. In this study, we observed a SDS-PAGE band that cross-reacted with anti-SaDyP2 antibody in the supernatant obtained from fragmented mycelia of $S$. avermitilis. In future, we will perform phenotypic studies following disruption of genes encoding SaDyP1 and SaDyP2 to clarify the physiological substrates and functions of these enzymes.

In conclusion, we heterologously expressed and characterized SaDyP1 from S. avermitilis. We hypothesize that SaDyP1 has a putative aspartate residue responsible for catalysis based on sequence and structure alignments. Moreover, SaDyP1 shows a lower optimal $\mathrm{pH}$, suggesting membership in the DyP-type peroxidase family. Typical substrates of peroxidases and anthraquinone dyes were oxidized by SaDyP1, although decolorizing rates were lower than those for DyP. Even though the results of this study demonstrated the expression of class V DyP-type peroxidases in S. avermitilis, there are no homologs of these proteins whose physiological substrates, functions, and tertiary structures have been elucidated; therefore, further study is warranted.

\section{Materials and Methods}

\subsection{Organisms and Materials}

S. avermitilis ATCC31267 was obtained from American Type Culture Collection (ATCC). pHSA81 and S. lividans were kindly provided by Dr. M. Kobayashi, University of Tsukuba. All chemical reagents and media used were analytical grade, unless otherwise specified.

\subsection{Plasmid Construction and S. lividans Transformation}

Plasmid construction and transformation procedures for expression of SaDyP1 in S. lividans were performed as previously described [19]. The gene encoding SaDyP1 was PCR-amplified from $S$. avermitilis genomic DNA using the primer pair $5^{\prime}$-GGA ATT CCA TAT G AGC ATC GAG AAG GGC-3' (forward) and 5'-GGA CTA GTT CAG TGG TGA TGG TGA TGA TGG GTG TCG AGG TCG GCG ATC CAG CGC AGC GCC GAC AGA CCG G-3' (reverse), and cloned as a $6 \times$ His-tagged protein into the pHSA81 expression vector for $S$. lividans. Each primer included a restriction enzyme site to facilitate cloning. S. lividans was transformed with pHSA81 harboring the amplified gene using polyethylene glycol (PEG)-mediated transformation of protoplasts, after which antibiotic-resistant transformants were selected by culturing with $1 \mu \mathrm{g} / \mathrm{mL}$ thiostrepton. 


\subsection{Purification of SaDyP1}

Transformed cells were cultured in $100 \mathrm{~mL}$ YEME medium ( $0.3 \%$ yeast extract, $0.5 \%$ Bacto-Tryptone, $0.3 \%$ malt extract, $1 \%$ glucose, $0.5 \%$ glycine, $5 \mathrm{mM} \mathrm{MgCl}_{2}, 34 \%$ sucrose, $\mathrm{pH}$ 7.0) at $29^{\circ} \mathrm{C}$ for 3 days. Cells were disrupted, and soluble protein in the supernatant was purified by Ni-NTA agarose affinity chromatography (Qiagen, Hilden, Germany) [19]. The eluted fraction was dialyzed against $25 \mathrm{mM}$ citrate buffer ( $\mathrm{pH}$ 5.5). In cases where addition of hemin chloride was necessary, hemin chloride was added at three molar equivalents relative to approximate protein content after dialysis. Following overnight incubation to incorporate heme, the solution containing the protein was dialyzed again to remove unincorporated hemin and applied to a Superdex 75pg column (bed volume, $120 \mathrm{~mL}$; Cytiva, Tokyo, Japan). Fractions ( $1 \mathrm{~mL}$ ) were collected, and fractions containing enzyme activity were pooled.

\subsection{Polyacrylamide Gel Electrophoresis, Immunoblotting and Spectral Characterization}

SDS-PAGE was performed as previously described [19]. After electrophoresis, proteins in gels were either stained with Coomassie Brilliant Blue G-250 (CBB-stain) or transferred to membranes for immunoblotting using polyclonal antibodies, produced against previously purified SaDyP2, used as an antigen. Spectral characteristics of purified SaDyP1 in $25 \mathrm{mM}$ citrate buffer ( $\mathrm{pH}$ 5.5) were assessed using a V-650 UV-Vis spectrophotometer (Jasco, Tokyo, Japan). Reinheit $Z a h l(\mathrm{Rz})$ value was calculated as $A_{406} / A_{280}$ ratio.

\subsection{Enzyme Assays and Characterization}

The optimum $\mathrm{pH}$ and thermostability of SaDyP1 were determined as previously described [19]. Briefly, SaDyP2 activities for ABTS, DMP and Acid Blue 324 were measured using a citrate buffer $(25 \mathrm{mM})$ covering a $\mathrm{pH}$ range of 3.0-5.5 and a phosphate buffer covering a $\mathrm{pH}$ range of 6.0-7.0. The assay solution consisted of $25 \mathrm{mM}$ buffer, $0.20 \mathrm{mM}$ $\mathrm{H}_{2} \mathrm{O}_{2}$ and $\sim 200 \mathrm{nM}$ SaDyP1.

The Michaelis-Menten constant $\left(K_{\mathrm{m}}\right)$ and catalytic constant $\left(k_{\text {cat }}\right)$ for ABTS and DMP were calculated in substrate-dependent enzymatic assay. The assay solution consisted of $25 \mathrm{mM}$ citrate buffer (pH 4.5 for Acid Blue 324 and pH 4.0 for other dyes), $0.20 \mathrm{mM} \mathrm{H}_{2} \mathrm{O}_{2}$ and $200 \mathrm{nM} \mathrm{SaDyP}$ and different concentrations of the substrates. The same conditions were measured three times, and the mean and standard deviation are shown. The data were fit to the Michaelis-Menten equation, Haldane equation or Hill equation. Kinetic parameters were calculated using molar absorption coefficients of $\varepsilon_{420}=36,000 \mathrm{~cm}^{-1} \mathrm{M}^{-1}$ for ABTS, $\varepsilon_{472}=49,600 \mathrm{~cm}^{-1} \mathrm{M}^{-1}$ for DMP, $\varepsilon_{608}=7700 \mathrm{~cm}^{-1} \mathrm{M}^{-1}$ for Acid Blue 324 .

\subsection{Decolorizing Rates for Anthraquinone and Azo Dyes}

The ability of SaDyP1 to degrade representative dyes containing anthraquinone or azo chromophores was also examined. The assay solution consisted of $25 \mathrm{mM}$ citrate buffer (pH 4.5 for Acid Blue 324 and $\mathrm{pH} 4.0$ for other dyes), $0.20 \mathrm{mM} \mathrm{H}_{2} \mathrm{O}_{2}$ and $200 \mathrm{nM} \mathrm{SaDyP}$, additionally containing $0.20 \mathrm{mM}$ Acid Blue $324\left(\varepsilon_{608}=7700 \mathrm{~cm}^{-1} \mathrm{M}^{-1}\right), 0.10 \mathrm{mM}$ AQ-2 $\left(\varepsilon_{600}=8300 \mathrm{~cm}^{-1} \mathrm{M}^{-1}\right), 0.10 \mathrm{mM}$ M303 $\left(\varepsilon_{476}=6200 \mathrm{~cm}^{-1} \mathrm{M}^{-1}\right), 0.02 \mathrm{mM}$ Reactive Black 5 $\left(\varepsilon_{598}=37,000 \mathrm{~cm}^{-1} \mathrm{M}^{-1}\right)$ or $0.03 \mathrm{mM}$ Reactive Red $33\left(\varepsilon_{500}=23,000 \mathrm{~cm}^{-1} \mathrm{M}^{-1}\right)$.

\subsection{Culture and Fractionation of S. avermitilis}

A suspension of S. avermitilis containing $2.0 \times 10^{9}$ spores was inoculated into $200 \mathrm{~mL}$ YEME medium without sucrose and incubated for $20 \mathrm{~h}$ at $30^{\circ} \mathrm{C}$ with shaking at $180 \mathrm{rpm}$. Mycelia from $30 \mathrm{~mL}$ cultures were collected by centrifugation at $7200 \times g$ for $30 \mathrm{~min}$. Collected mycelia were disrupted three times at $2.0 \times 10^{4} \mathrm{psi}$ using a French press (Otake Seisakusyo) and centrifuged for $30 \mathrm{~min}$ at $7200 \times g$ to pellet cell debris. The precipitates were suspended in $1 \mathrm{~mL}$ of phosphate buffer and solubilized by incubating overnight with an equal volume of $10 \%$ SDS. An equal volume of $50 \%$ glycerol was added to the supernatant, and the total volume was concentrated $\sim 40$-fold by ultrafiltration (Amicon 
Ultra-15 30 K; Merck Millipore, Burlington, USA). Proteins in the concentrated supernatant were resolved by electrophoresis using $12 \mu \mathrm{L}$ of supernatant per lane.

Author Contributions: K.S.; investigation, data curation, methodology, writing-original draft. T.Y.; investigation, formal analysis, methodology, writing - review and editing. R.H.; investigation. R.T.; investigation. H.A.; investigation Y.K.; investigation. Y.I.; investigation. Y.S. conceptualization, funding acquisition, resources, writing-review and editing, supervision. All authors have read and agreed to the published version of the manuscript.

Funding: This work was performed under the Cooperative Research Program of "NJRC Mater. \& Dev." Institutional Review Board Statement: Not applicable.

Informed Consent Statement: Not applicable.

Data Availability Statement: Data sharing is not applicable to this article.

Acknowledgments: We are grateful to M. Kobayashi, University of Tsukuba, for kindly providing pHSA81 and S. lividans. We would like to thank M. Morita, Tokyo Institute of Technology, for help to construct transformant.

Conflicts of Interest: The authors declare no conflict of interest. The funders had no role in the design of the study; in the collection, analysis or interpretation of data; in the writing of the manuscript or in the decision to publish the results.

\section{References}

1. Fawal, N.; Li, Q.; Savelli, B.; Brette, M.; Passaia, G.; Fabre, M.; Mathé, C.; Dunand, C. PeroxiBase: A database for large-scale evolutionary analysis of peroxidases. Nucleic Acids Res. 2013, 41, D441-D444. [CrossRef]

2. Savelli, B.; Li, Q.; Webber, M.; Jemmat, A.M.; Robitaille, A.; Zamocky, M.; Mathe, C.; Dunand, C. RedoxiBase: A database for ROS homeostasis regulated proteins. Redox Biol. 2019, 26, 101247. [CrossRef]

3. Welinder, K.G. Superfamily of plant, fungal and bacterial peroxidases. Curr. Opin. Struct. Biol. 1992, 2, 388-393. [CrossRef]

4. Kim, S.J.; Shoda, M. Purification and characterization of a novel peroxidase from Geotrichum candidum Dec 1 involved in decolorization of dyes. Appl. Environ. Microbiol. 1999, 65, 1029-1035. [CrossRef]

5. Sugano, Y.; Nakano, R.; Sasaki, K.; Shoda, M. Efficient heterologous expression in Aspergillus oryzae of a unique dye-decolorizing peroxidase, DyP, of Geotrichum candidum. Appl. Environ. Microbiol. 2000, 66, 1754-1758. [CrossRef] [PubMed]

6. Sugano, Y.; Muramatsu, R.; Ichiyanagi, A.; Sato, T.; Shoda, M. DyP, a unique dye-decolorizing peroxidase, represents a novel heme peroxidase family: ASP171 replaces the distal histidine of classical peroxidases. J. Biom. Chem. 2007, 282, 36652-36658. [CrossRef]

7. Sugano, Y. DyP-type peroxidases comprise a novel heme peroxidase family. Cell Mol. Life Sci. 2009, 66, 1387-1403. [CrossRef] [PubMed]

8. Yoshida, T.; Sugano, Y. A structural and functional perspective of DyP-type peroxidase family. Arch. Biochem. Biophys. 2015, 574, 49-55. [CrossRef] [PubMed]

9. Johjima, T.; Ohkuma, M.; Kudo, T. Isolation and cDNA cloning of novel hydrogen peroxide-dependent phenol oxidase from the basidiomycete Termitomyces albuminosus. Appl. Microbiol. Biotechnol. 2003, 61, 220-225. [CrossRef]

10. Liers, C.; Bobeth, C.; Pecyna, M.; Ullrich, R.; Hofrichter, M. DyP-like peroxidases of the jelly fungus Auricularia auricula-judae oxidize nonphenolic lignin model compounds and high-redox potential dyes. Appl. Microbiol. Biotechnol. 2010, 85, 1869-1879. [CrossRef] [PubMed]

11. Scheibner, M.; Hülsdau, B. Novel peroxidases of Marasmius scorodonius degrade $\beta$-carotene. Appl. Microbiol. Biotechnol. 2008, 85, 1241-1250. [CrossRef]

12. Salvachúa, D.; Prieto, A.; Martínez, Á.T.; Martínez, M.J. Characterization of a novel dye-decolorizing peroxidase (DyP)-type enzyme from Irpex lacteus and its application in enzymatic hydrolysis of wheat straw. Appl. Environ. Microbiol. 2013, 79, 4316-4324. [CrossRef] [PubMed]

13. Liers, C.; Pecyna, M.J.; Kellner, H.; Worrich, A.; Zorn, H.; Steffen, K.T.; Hofrichter, M.; Ullrich, R. Substrate oxidation by dye-decolorizing peroxidases (DyPs) from wood- and litter-degrading agaricomycetes compared to other fungal and plant heme-peroxidases. Appl. Microbiol. Biotechnol. 2013, 97, 5839-5849. [CrossRef] [PubMed]

14. Sugawara, K.; Igeta, E.; Amano, Y.; Hyuga, M.; Sugano, Y. Degradation of antifungal anthraquinone compounds is a probable physiological role of DyP secreted by Bjerkandera adusta. AMB Express 2019, 9, 56. [CrossRef] [PubMed]

15. Brown, M.E.; Barros, T.; Chang, M.C.Y. Identification and Characterization of a Multifunctional Dye Peroxidase from a LigninReactive Bacterium. ACS Chem. Biol. 2012, 7, 2074-2081. [CrossRef]

16. Ogola, H.J.O.; Kamiike, T.; Hashimoto, N.; Ashida, H.; Ishikawa, T.; Shibata, H.; Sawa, Y. Molecular characterization of a novel peroxidase from the cyanobacterium Anabaena sp. strain PCC 7120. Appl. Environ. Microbiol. 2009, 75, 7509-7518. [CrossRef] 
17. Ogola, H.J.O.; Hashimoto, N.; Miyabe, S.; Ashida, H.; Ishikawa, T.; Shibata, H.; Sawa, Y.; Joseph, H.; Ogola, O.; Hashimoto, N. Enhancement of hydrogen peroxide stability of a novel Anabaena sp. DyP-type peroxidase by site-directed mutagenesis of methionine residues. Appl. Microbiol. Biotechnol. 2010, 87, 1727-1736. [CrossRef] [PubMed]

18. Yoshida, T.; Ogola, H.J.O.; Amano, Y.; Hisabori, T.; Ashida, H.; Sawa, Y.; Tsuge, H.; Sugano, Y. Anabaena sp. DyP-type peroxidase is a tetramer consisting of two asymmetric dimers. Proteins: Struct. Funct. Bioinform. 2016, 84, 31-42. [CrossRef]

19. Sugawara, K.; Nishihashi, Y.; Narioka, T.; Yoshida, T.; Morita, M.; Sugano, Y. Characterization of a novel DyP-type peroxidase from Streptomyces avermitilis. J. Biosci. Bioeng. 2017, 123, 425-430. [CrossRef]

20. Létoffé, S.; Heuck, G.; Delepelaire, P.; Lange, N.; Wandersman, C. Bacteria capture iron from heme by keeping tetrapyrrol skeleton intact. Proc. Natl. Acad. Sci. USA 2009, 106, 11719-11724. [CrossRef]

21. Dailey, H.A.; Septer, A.N.; Daugherty, L.; Thames, D.; Gerdes, S.; Stabb, E.V.; Dunn, A.K. The Escherichia coli Protein YfeX Functions as a Porphyrinogen Oxidase. Not Heme Dechelatase 2011, 2, 1-8.

22. Cao, J.; Woodhall, M.R.; Alvarez, J.; Cartron, M.L.; Andrews, S.C. EfeUOB (YcdNOB) is a tripartite, acid-induced and CpxARregulated, low- $\mathrm{pH} \mathrm{Fe}^{2+}$ transporter that is cryptic in Escherichia coli K-12 but functional in E. coli O157:H7. Mol. Microbiol. 2007, 65, 857-875. [CrossRef] [PubMed]

23. Große, C.; Scherer, J.; Koch, D.; Otto, M.; Taudte, N.; Grass, G. A new ferrous iron-uptake transporter, EfeU (YcdN), from Escherichia coli. Mol. Microbiol. 2006, 62, 120-131. [CrossRef]

24. Rajasekaran, M.B.; Nilapwar, S.; Andrews, S.C.; Watson, K.A. EfeO-cupredoxins: Major new members of the cupredoxin superfamily with roles in bacterial iron transport. BioMetals 2010, 23, 1-17. [CrossRef] [PubMed]

25. Ollinger, J.; Song, K.B.; Antelmann, H.; Hecker, M.; Helmann, J.D. Role of the Fur regulon in iron transport in Bacillus subtilis. J. Bacteriol. 2006, 188, 3664-3673. [CrossRef]

26. Ikeda, H.; Ishikawa, J.; Hanamoto, A.; Shinose, M.; Kikuchi, H.; Shiba, T.; Sakaki, Y.; Hattori, M.; Omura, S. Complete genome sequence and comparative analysis of the industrial microorganism Streptomyces avermitilis. Nat. Biotechnol. 2003, 21, 526-531. [CrossRef] [PubMed]

27. Petrus, M.L.C.; Vijgenboom, E.; Chaplin, A.K.; Worrall, J.A.R.; van Wezel, G.P.; Claessen, D. The DyP-type peroxidase DtpA is a Tat-substrate required for GlxA maturation and morphogenesis in Streptomyces. Open Biol. 2016, 6, 150149. [CrossRef]

28. Kekilli, D.; Moreno-Chicano, T.; Chaplin, A.K.; Horrell, S.; Dworkowski, F.S.N.; Worrall, J.A.R.; Strange, R.W.; Hough, M.A Photoreduction and validation of haem-ligand intermediate states in protein crystals by in situ single-crystal spectroscopy and diffraction. IUCrJ 2017, 4, 263-270. [CrossRef]

29. Chaplin, A.K.; Wilson, M.T.; Worrall, J.A.R. Kinetic characterisation of a dye decolourising peroxidase from Streptomyces lividans: New insight into the mechanism of anthraquinone dye decolourisation. Dalton Trans. 2017, 46, 9420-9429. [CrossRef]

30. Chaplin, A.K.; Chicano, T.M.; Hampshire, B.V.; Wilson, M.T.; Hough, M.A.; Svistunenko, D.A.; Worrall, J.A.R. An Aromatic Dyad Motif in Dye Decolourising Peroxidases Has Implications for Free Radical Formation and Catalysis. Chem. Eur. J. 2019, 25, 6141-6153. [CrossRef]

31. Lucic, M.; Chaplin, A.K.; Moreno-Chicano, T.; Dworkowski, F.S.N.; Wilson, M.T.; Svistunenko, D.A.; Hough, M.A.; Worrall, J.A.R. A subtle structural change in the distal haem pocket has a remarkable effect on tuning hydrogen peroxide reactivity in dye decolourising peroxidases from Streptomyces lividans. Dalton Trans. 2020, 49, 1620-1636. [CrossRef]

32. Yoshida, T.; Tsuge, H.; Konno, H.; Hisabori, T.; Sugano, Y. The catalytic mechanism of dye-decolorizing peroxidase DyP may require the swinging movement of an aspartic acid residue. FEBS J. 2011, 278, 2387-2394. [CrossRef]

33. Sugano, Y.; Matsushima, Y.; Tsuchiya, K.; Aoki, H.; Hirai, M.; Shoda, M. Degradation pathway of an anthraquinone dye catalyzed by a unique peroxidase DyP from Thanatephorus cucumeris Dec 1. Biodegradation 2009, 20, 433-440. [CrossRef] [PubMed]

34. Haldane, J.B.S. Enzymes; Longmans, Green: London, UK, 1930.

35. Yoshida, T.; Tsuge, H.; Hisabori, T.; Sugano, Y. Crystal structures of dye-decolorizing peroxidase with ascorbic acid and 2,6-dimethoxyphenol. FEBS Lett. 2012, 586, 4351-4356. [CrossRef] [PubMed]

36. Komatsu, M.; Uchiyama, T.; Omura, S.; Cane, D.E.; Ikeda, H. Genome-minimized Streptomyces host for the heterologous expression of secondary metabolism. Proc. Natl. Acad. Sci. USA 2010, 107, 2646-2651. [CrossRef] [PubMed]

37. Mendes, S.; Catarino, T.; Silveira, C.; Todorovica, S.; Martinsa, L.O. Catalytic mechanism of BsDyP an A-type dye-decolourising peroxidase: Neither aspartate nor arginine is individually essential for peroxidase activity. Catal. Sci. Technol. 2015, 5, 5196-5207. [CrossRef]

38. Liu, X.; Du, Q.; Wang, Z.; Zhu, D.; Huang, Y.; Li, N.; Wei, T.; Xu, S.; Gu, L. Crystal Structure and Biochemical Features of EfeB/YcdB from Escherichia coli O157. J. Biol. Chem. 2011, 286, 14922-14931. [CrossRef]

39. Singh, R.; Grigg, J.C.; Armstrong, Z.; Murphy, M.E.; Eltis, L.D. Distal Heme Pocket Residues of B-type Dye-decolorizing Peroxidase. J. Biol. Chem. 2012, 287, 10623-10630. [CrossRef] [PubMed]

40. Santos, A.; Mendes, S.; Brissos, V.; Martins, L.O. New dye-decolorizing peroxidases from Bacillus subtilis and Pseudomonas putida MET94: Towards biotechnological applications. Appl. Microbiol. Biotechnol. 2014, 98, 2053-2065. [CrossRef]

41. Van Bloois, E.; Torres Pazmino, D.E.; Winter, R.T.; Fraaije, M.W. A robust and extracellular heme-containing peroxidase from Thermobifida fusca as prototype of a bacterial peroxidase superfamily. Appl. Microbiol. Biotechnol. 2010, 86, 1419-1430. [CrossRef] 
42. Rha, R.; Roberts, J.N.; Singh, R.; Grigg, J.C.; Murphy, M.E.P.; Bugg, T.D.H.; Eltis, L.D. Characterization of Dye-Decolorizing Peroxidases from Rhodococcus jostii RHA1. Biochemistry 2011, 50, 5108-5119.

43. Uchida, T.; Omura, I.; Umetsu, S.; Ishimori, K. Radical transfer but not heme distal residues is essential for $\mathrm{pH}$ dependence of dye-decolorizing activity of peroxidase from Vibrio cholera. J. Inorg. Biochem. 2021, 219, 111422. [CrossRef] [PubMed] 